Anaesthesist $2005 \cdot 54: 8-16$ DOI 10.1007/s00101-004-0794-x

Online publiziert: 18. Dezember 2004

c) Springer Medizin Verlag 2004

Redaktion

K. Peter, München
E.-M. Jordi Ritz - T. O. Erb - F. J. Frei

Universitäts-Kinderspital beider Basel (UKBB), Basel, Schweiz

\title{
Vaskulärer Zugang in der Kindernotfallanästhesie
}

Ein intravenöser Zugang ist bei Säuglingen und Kindern schwieriger zu legen als beim Erwachsenen. Diese Gegebenheit kann bei Kinderanästhesiologischen Notfällen eine zentrale Bedeutung bekommen, weil sich die intravenöse Blitzeinleitung als die sicherste Methode zum effektiven Schutz der Atemwege vor einer Aspiration etabliert hat. Anatomische Besonderheiten erlauben es, beim Kind intravenöse Zugänge an Stellen anzubringen, die bei Erwachsenen unüblich sind, z. B. am Kopf oder an den Füßen. Wenn ein intravenöser Zugang nicht möglich ist, muss unter Berücksichtigung der vorhandenen Risiken zwischen der intraossären, intramuskulären oder inhalativen Anästhesieeinleitung gewählt werden.

Gewiss kennen alle Anästhesisten den Seitenblick während des Prämedikationsgespräches auf die Handrücken des gut genährten Säuglings (• Abb. 1), dem zur notfallmäßigen Anästhesieeinleitung ein i.v.-Zugang angelegt werden soll. Wie kann weiter vorgegangen werden, wenn für die Blitzeinleitung beim aspirationsgefährdeten Kind die Punktion einer peripheren Vene misslingt?

Das Anlegen eines vaskulären Zugangs ist neben dem Atemwegsmanagement eine der elementarsten Aufgaben der anästhesiologischen Tätigkeit. Insbesondere bei Kleinkindern werden häufig schwierige Punktionsverhältnisse angetroffen [22, 33]. Neben den ohnehin schon kleineren anatomischen Verhältnissen und der gröBeren Anreicherung subkutanen Fettgewe- bes erschweren die verminderte Kooperation des Kindes und der hohe Erfolgsdruck, im Beisein verängstigter Eltern atraumatisch einen intravenösen Zugang zu legen, die Punktion zusätzlich.

Die gängige Lehrmeinung zur anästhesiologischen Notfallversorgung nicht nüchterner Kleinkinder empfiehlt die endotracheale Intubation unter den Bedingungen einer Blitzeinleitung. Dafür ist jedoch das Legen eines intravenösen Zugangs im Wachzustand unabdingbar. Kann dies nicht ohne weiteres erfolgen, stellt sich die Frage, welche weiteren vaskulären Zugänge zur Verfügung stehen, wenn das Aspirationsrisiko für eine Anästhesieeinleitung ohne einen intravenösen Zugang als zu hoch betrachtet wird. Inwieweit besteht beim nicht nüchternen Kleinkind die Möglichkeit eines Anästhesievorgehens ohne einen vaskulären Zugang? Die Praktikabilität und das Risiko von Alternativverfahren für ein solches Vorgehen sollen thematisiert werden.

Einfache Antworten zum Vorgehen bei schwierigen Venenverhältnissen sind nicht möglich, und wissenschaftliche Daten stehen dazu kaum zur Verfügung. Mithilfe einer Umfrage wurde deshalb versucht, die verschiedenen Ansichten und Vorgehensweisen erfahrener Anästhesisten, die ausreichend Praxis im Umgang mit Kleinkindern aufweisen, zu erfassen, um daraus gemeinsame Gedanken und mögliche Empfehlungen zu erarbeiten.

\section{Umfrage}

An die Leiter der Weiterbildungsstätten für Anästhesie in der Schweiz und an alle Mitglieder der Schweizerischen Gesell- schaft für Kinderanästhesie wurden insgesamt 89 Fragebögen verschickt. Die Empfänger wurden gebeten, mithilfe der folgenden 2 Fallbeispiele Fragen zu beantworten und ihre eigenen Erfahrungen im Umgang mit schwierigen Punktionsverhältnissen zu dokumentieren.

\section{Fall A: mäßige Aspirationsgefahr}

Ein 3-jähriges Kleinkind stürzte vor einer Stunde von der Schaukel. Es zog sich eine Vorderarmfraktur $z u$, die operativ versorgt werden muss. Das Kind ist nicht nüchtern. Der Chirurg drängt zur baldigen Intervention, da neurologische Ausfälle vorhanden

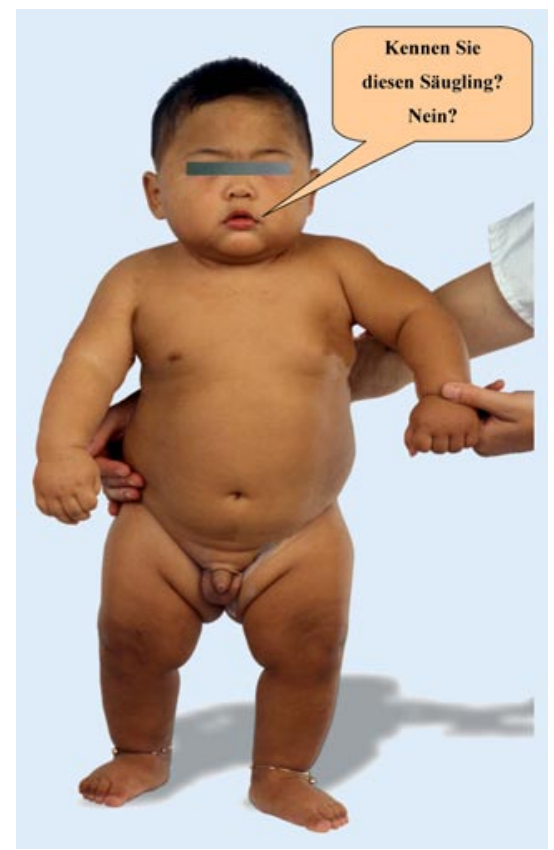

Abb. $1 \triangle$ Schwierige Venenverhältnisse können in jeder Alterskategorie vorhanden sein. Gehäuft treten sie jedoch bei Säuglingen nach der Neugeborenenperiode auf 
Tabelle 1

Häufigkeit der gewählten Möglichkeiten mit vaskulärem Zugang

\begin{tabular}{|lll|}
\hline & $\begin{array}{l}\text { Fallbeispiel A }(\boldsymbol{n}=\mathbf{5 4}) \\
\text { Anzahl Anästhesisten }\end{array}$ & $\begin{array}{l}\text { Fallbeispiel B }(\boldsymbol{n}=\mathbf{4 8}) \\
\text { Anzahl Anästhesisten }\end{array}$ \\
\hline Weitere periphervenöse Punktionsversuche & 41 (76) & $35(73)$ \\
\hline Skalpvenen & 12 & 32 \\
\hline V. jugularis externa & 0 & 9 \\
\hline Zentralvenenpunktion & 0 & $10(21)$ \\
\hline V. femoralis & 0 & 5 \\
\hline V. subclavia & 0 & 4 \\
\hline V. jugularis interna & 0 & 1 \\
\hline Intraossärnadel & 0 & $23(48)$ \\
\hline Venenfreilegung & 0 & $14(29)$ \\
\hline
\end{tabular}

Werte in Klammern Angaben in Prozent

Tabelle 2

Häufigkeit der gewählten Möglichkeiten ohne vaskulären Zugang

Einleitungen

Intramuskulär

Inhalativ

Rektal
Fallbeispiel A ( $n=54)$ Anzahl Anästhesisten [\%] 59 50

7
Fallbeispiel B $(n=48)$ Anzahl Anästhesisten [\%] 33 25 sind. Eine intravenöse Blitzeinleitung ist geplant. Bereits wurde 2-mal vergebens versucht, dem Kind einen venösen Zugang zu legen.

\section{Fall B: hohe Aspirationsgefahr}

Ein 10 Monate alter Säugling wurde vor 7 Tagen wegen einer Duodenalstenose laparotomiert. Er zeigt klinisch die Zeichen eines Ileus, erbricht, ist ansonsten aber lebhaft. Zur Relaparotomie und präoperativen Rehydrierung wird ein venöser Zugang benötigt. Bisher wurde 3-mal versucht, eine periphere Vene zu punktieren, ohne Erfolg. Das Kind erbricht nochmals und schreit.

\section{Fragen zum konkreten Vorgehen in den Fallbeispielen}

I. Welche vaskulären Zugänge haben nach gescheiterten peripheren Punktionsversuchen in der Praxis Priorität, wenn das Aspirationsrisiko für ein Vorgehen ohne vaskulären Zugang als zu hoch betrachtet wird?

II. Welche Möglichkeiten bestehen, die Anästhesie beim nicht nüchternen
Kleinkind ohne vaskulären Zugang einzuleiten?

\section{Fragen zu eigenen Erfahrungen mit dem schwierigen vaskulären Zugang und der intraossären Punktion}

III. Kennen Sie die Situation, ein aspirationsgefährdetes Kind nicht intravenös eingeleitet zu haben, weil der Zugang nicht gelegt werden konnte?

IV. Zu welcher Indikation haben Sie schon einen intraossären Zugang gelegt?

\section{Resultate}

Von 79 Befragten erfolgte eine schriftliche oder telefonische Antwort. Hierbei gaben 25 Personen an, im Rahmen ihrer gegenwärtigen Tätigkeit nicht oder nicht mehr mit Kinderanästhesie konfrontiert zu sein. Für die Auswertung liegen somit die Antworten von 54 Anästhesiefachärzten mit einer Berufspraxis von durchschnittlich 18,4 Jahren (43 Chef- oder leitende Ärzte, 8 Oberärzte und 3 Fachärzte) vor.
Antworten zum konkreten Vorgehen in den Fallbeispielen

Zum Fall A konnten alle 54 Fragebögen in die Auswertung einbezogen werden. In Fall B waren es 48 Antworten. Weil in ihrer Klinik Kinder in einem Alter unter einem Jahr nicht anästhesiert werden, nahmen 6 Anästhesisten keine Stellung. Die verbleibenden 48 Befragten verfügen über regelmäßige Praxis in der Säuglings- und Kleinkindanästhesie (je mindestens 3 Patienten/Woche).

\section{Vorgehen mit vaskulärem Zugang} Am häufigsten werden weitere periphervenöse Punktionsversuche von über $70 \%$ der Befragten in beiden Fallbeispielen in Betracht gezogen (• Tabelle 1). In Fall A würden durchschnittlich noch 4, in Fall B 5 weitere Punktionsversuche unternommen. Dabei gehört die Punktion der V. jugularis externa und der Skalpvenen zur gängigen Praxis. Letztere wird jedoch nur beim Säugling angewendet. Spätestens nach dem Scheitern einiger Punktionsversuche sehen über 90\% der Anästhesisten eine rektale Prämedikation mit Midazolam vor. Ein Anästhesist erwähnt die Venenpunktion unter Analgosedation eines LachgasSauerstoff- $\left(\mathrm{N}_{2} \mathrm{O}-\mathrm{O}_{2}-\right)$ Gemisches für die Situation des nicht nüchternen Kleinkindes.

Bei hoher Aspirationsgefahr (Fallbeispiel B) schlagen $21 \%$ der Befragten die Punktion einer zentralen Vene vor. Beinahe mit gleicher Häufigkeit wird dazu der femorale Zugang oder die infraklavikuläre Kanülierung der V. subclavia gewählt. Die primäre Punktion der V. jugularis interna stellt nur für einen einzelnen Anästhesisten ein möglicher Zugang dar. Wenn andere Techniken nicht zum Erfolg führen, gilt der Einsatz einer Intraossärnadel als mögliche Alternative bei $48 \%$ der Befragten. Schließlich wird bei Fall B in 31\% der Fälle die chirurgische Freilegung einer Vene in Lokalanästhesie in Betracht gezogen.

\section{Vorgehen ohne vaskulären Zugang}

Bei mäßiger Aspirationsgefahr (Fall A) stehen die intramuskuläre Anästhesieeinleitung mit Ketamin und die inhalative Einleitung mit einem volatilen Anästhetikum als Vorgehensoptionen gleichermaßen im Vordergrund. 
Anaesthesist $2005 \cdot 54: 8-16$

DOI 10.1007/s00101-004-0794-x

๑) Springer Medizin Verlag 2004

\section{E.-M. Jordi Ritz · T. O. Erb · F. J. Frei}

\section{Vaskulärer Zugang in der Kindernotfallanästhesie}

\section{Zusammenfassung}

Zum Thema des schwierigen intervenösen

Zugangs bei pädiatrischen Notfallsituationen existieren erstaunlich wenige Angaben in der Literatur.,"Wie machen es die Anderen?" war die Motivationsgrundlage für eine Umfrage bei in Kinderanästhesie erfahrenen Anästhesisten.

Insgesamt 89 Fragebögen wurden an die Leiter der Weiterbildungsstätten für Anästhesie in der Schweiz und an alle Mitglieder der Schweizerischen Gesellschaft für Kinderanästhesie verschickt. Anhand von 2 Fallbeispielen (Fall A: nicht nüchternes Kleinkind mit einer Radiusfraktur, Fall B: Säugling mit hohem lleus) wurde das weitere Vorgehen nach 2-3 erfolglosen peripheren Punktionsversuchen erfragt. Die Beantwortung ergab, dass die meisten der Befragten in beiden Situationen zunächst wei- tere periphere Venenpunktionen vornehmen werden. Falls diese Versuche erfolglos bleiben, wird beim Kleinkind mit der Radiusfraktur eine intramuskuläre oder inhalative Anästhesieeinleitung befürwortet. Bei dem Säugling mit lleus wird versucht, für die Anästhesieeinleitung einen intraossären oder zentralvenösen Zugang (V. femoralis) zu legen. Aufgrund der Resultate der Umfrage und einer Literaturrecherche wird eine Prioritätenliste zu den wichtigsten vaskulären Zugängen und alternativen Anästhesieeinleitungsmethoden in der pädiatrischen Notfallsituation vorgeschlagen.

\section{Schlüsselwörter}

Kinderanästhesie · Notfall ·

Komplikationen · Vaskulärer Zugang ·

Alternative Anästhesieeinleitung

\section{Vascular access in emergency paediatric anaesthesia}

\section{Abstract}

There is very little literature to guide the young practitioner in caring for a child that needs emergency surgery and has difficult venous access.

Questionnaires were sent to 89 members of the Swiss Paediatric Anaesthesia Society and to the heads of Anaesthesia Departments of Swiss teaching hospitals. Two typical case records were presented, both of which were characterised by the fact that 2-3 peripheral venous cannulation attempts were unsuccessful. Case A: a young child with a fracture of the radius and case $B$ an infant with upper gastrointestinal ileus. The anaesthetists were then questioned regarding their preferences for optimal treatment. The majority would pro- ceed with further attempts and, if these still failed, intramuscular or inhalational induction of anaesthesia was suggested as a reasonable choice for case $A$. However, for case $B$, a femoral venous or intraosseous access to the venous system was judged to be the safest method. On the basis of our inquiry and a literature search, a priority list was developed to suggest the best possible techniques for vascular access and alternative anaesthesia induction techniques for emergency paediatric procedures.

\section{Keywords}

Paediatric anaesthesia - Emergency . Complications - Vascular access . Alternative anaesthesia procedures
Die intramuskuläre oder inhalative Einleitung wurde bei hoher Aspirationsgefahr (Fall B) deutlich seltener als gangbare Maßnahme in Betracht gezogen ( $\bullet$ Tabelle 2).

Selten wird eine rektale Anästhesieeinleitung erwähnt, hierbei wird die Applikation von Ketamin, Thiopental, Methohexital oder eine Kombination von Thiopental und Fentanyl vorgeschlagen. Von den Anästhesisten wählen 4\% ein regionales Anästhesieverfahren ohne vaskulären Zugang.

Die zur Auswahl stehenden Möglichkeiten wurden von den befragten Anästhesisten zudem nach ihrer Priorität geordnet. Die Erfassung des konkreten Vorgehens in den beschriebenen Fallbeispielen dient zur Erstellung einer Prioritätenliste im Umgang mit aspirationsgefährdeten Kin$\operatorname{dern}(\bullet$ Abb. 2).

Antworten zu eigenen Erfahrungen im Umgang mit dem schwierigen vaskulären Zugang und der intraossären Punktion

Die Antworten aller 54 Anästhesisten konnten in die Auswertung einbezogen werden.

\section{Umgang mit dem schwierigen vaskulären Zugang}

Von den Befragten geben $63 \%$ an, mit der Situation vertraut zu sein, ein Kind ohne vaskulären Zugang einzuleiten. Es erinnern sich 22\% der Anästhesisten konkret an die Situation, bei einem Kind mit Ileus dieses Verfahren nach erfolglosen Versuchen gewählt zu haben; hierbei wird meistens der intramuskulären Technik gegenüber der inhalativen der Vorzug gegeben.

\section{Umgang mit der intraossären Punktion}

In der Praxis schon einen oder mehrere intraossäre Zugänge gelegt zu haben, geben $63 \%$ der Anästhesisten an. Dies geschah meist im Rahmen einer Reanimation oder Hypovolämiebehandlung, selten zur Anästhesieeinleitung. Selbst in der Reanimationssituation wurde nur in $12 \% \mathrm{der}$ Fälle primär intraossär punktiert; 59\% der Anästhesisten haben vorerst mehrere periphere, $29 \%$ zusätzlich zentralvenöse Punktionsversuche unternommen. 


\section{Management bei schwierigen Venenverhältnissen}

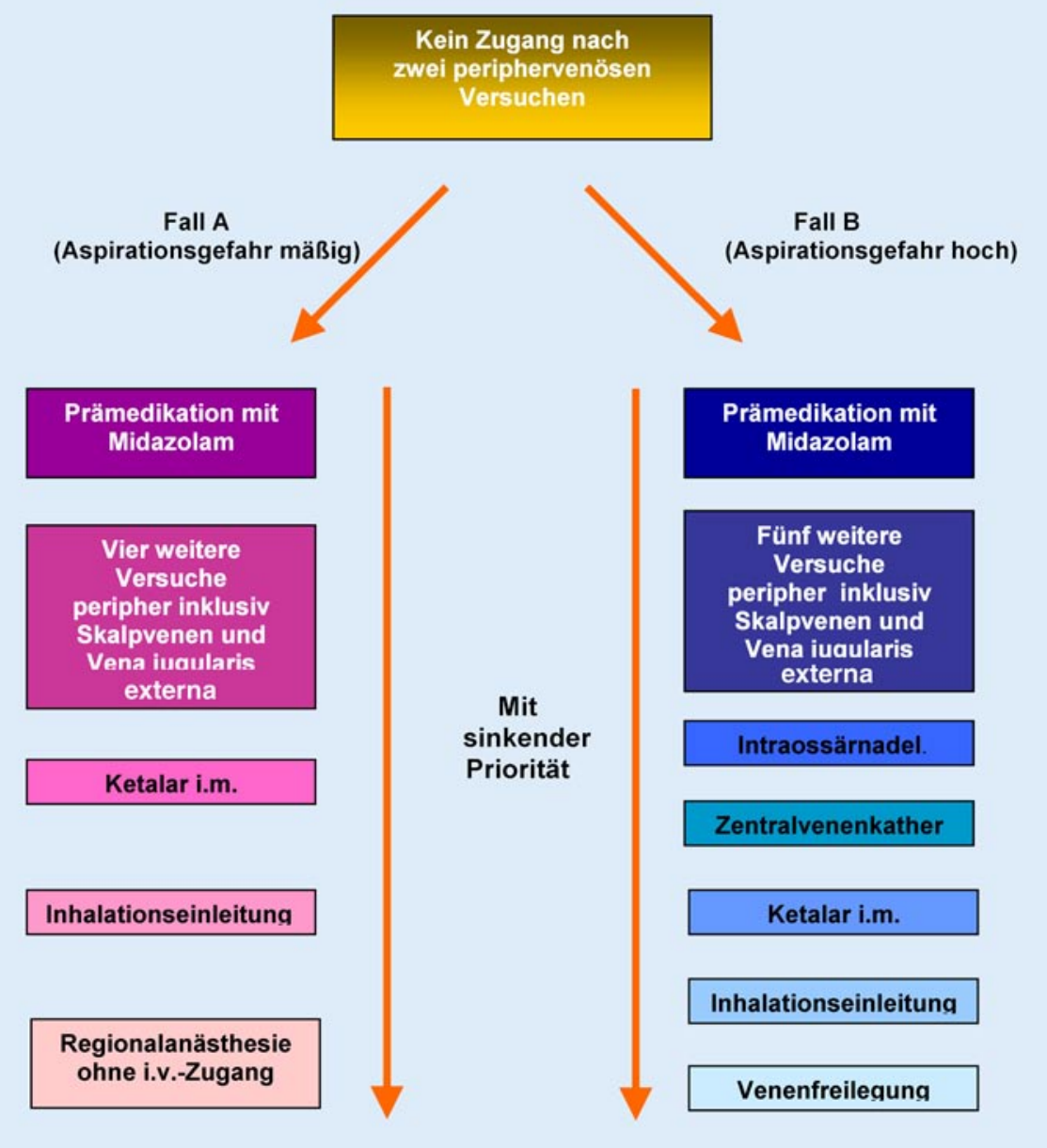

Abb. $2 \Delta$ Aus den Antworten der Umfrage erstellte Prioritätenliste für das weitere Vorgehen bei 2 typischen pädiatrischen Notfällen (Fall A und B; s. Text), die wegen erhöhtem Aspirationsrisiko eine Blitzeinleitung benötigen

\section{Diskussion}

Für viele Tätigkeiten in der Anästhesie gibt es Algorithmen, die insbesondere in Notfallsituationen mithelfen, das Vorgehen aufgrund von rationalen Argumenten hierarchisch zu gliedern. Der Umgang mit schwierigen Venenpunktionen ist eines der zentralen Themen der Kinderanästhesie. Für die Reanimationssituation existieren dazu Richtlinien, Empfehlungen und Algorithmen. Bei den 2 vorgestellten Fällen soll aber nicht das Vorgehen unter Reanimationsbedingungen diskutiert werden, sondern die Abfolge der Maßnahmen in der viel häufiger auftretenden dringlichen Situation, in der das nicht nüchterne Kind eines vaskulären Zugangs zur Anästhesieeinleitung bedarf.

Die Gliederung der Diskussion lehnt sich an die aus der Auswertung der Um- frage erstellten Prioritätenliste (• Abb. 2). Diese zeigt lediglich eine statistische $\mathrm{Zu}$ sammenfassung der Befragung auf. In Abwesenheit von wissenschaftlichen Daten zum Thema hat diese Vorgehensweise den Vorteil, die Meinung von Anästhesisten mit unterschiedlicher Erfahrung in Kinderanästhesie aus Peripherie- und Zentrumskliniken der Schweiz zu erfassen. Die Aussagekraft der Resultate ist beschränkt. Es lässt sich daraus nicht ableiten, welche der Methoden das geringste Risiko in sich birgt.

\section{Prämedikation}

Über 90\% der befragten Anästhesisten prämedizieren das Kind nach erfolglosen Punktionsversuchen mit Midazolam. Midazolam hat keinen Effekt auf Volumen und $\mathrm{pH}$ des Magensaftes [28]. Es bewirkt lediglich Anxiolyse, Amnesie und Sedation. Man kann davon ausgehen, dass die Stressantwort vermindert wird und damit das Aspirationsrisiko evtl. reduziert werden kann. Die Möglichkeit, für die Kanülierung eine Analgosedation mit einem $\mathrm{N}_{2} \mathrm{O}-\mathrm{O}_{2}$-Gemisch durchzuführen, wird von einem der Anästhesisten als Option erwähnt. Die Lachgasinhalation wird für schmerzhafte Prozeduren bei pädiatrischen Notfällen beschrieben [15], für die Anwendung beim explizit nicht nüchternen Kind fehlen die Angaben.

Nur wenige Autoren setzen eine pharmakologische Prämedikation mit Säureblockern oder Prokinetika ein. Diese reduzieren Menge und Säure des Mageninhalts bzw. beschleunigen die Magenentleerung. Keine Studie konnte bislang jedoch eine damit verbundene Effektivität zur Reduktion der Inzidenz oder der Konsequenzen einer Aspiration aufzeigen [11]. Die Kosten dieser Maßnahme scheinen in Anbetracht des fehlenden Nutzens nicht gerechtfertigt.

\section{Vorgehen mit vaskulärem Zugang}

\section{Peripherer Venenzugang}

In der hierarchischen Abfolge des Managements des aspirationsgefährdeten Kindes ist der periphere Venenzugang die primäre Intervention. Typische Punktionsstellen sind die Venen des Hand- und Fußrückens. Venen an anderen typischen Lokalisationen sind in vielen Fällen nur schlecht oder überhaupt nicht sichtbar, sind aber häufig gut palpabel (V. saphena magna vor dem medialen Malleolus, V. cephalica und V. basilica in der Fossa cubita). Da die Lokalisation dieser Venen relativ konstant ist, werden sie gelegentlich auch erfolgreich „blind“ punktiert.

$\mathrm{Zu}$ den favorisierten Punktionsorten bei schwierigen Venenverhältnissen gehören bei Säuglingen die praktisch immer sichtbaren Skalpvenen. Durch die bindegewebige Verankerung im Knochen ist ein Kollabieren ihres Lumens nahezu unmöglich. Das Anstechen der Vene erfolgt gegen kaudal, in Richtung des venösen $\mathrm{Ab}$ flusses.

Die Punktion der V. jugularis externa mit einer kurzen Verweilkanüle wird von vielen Praktikern als eine gute Alternative betrachtet. Der Kopf des Kindes wird un- 


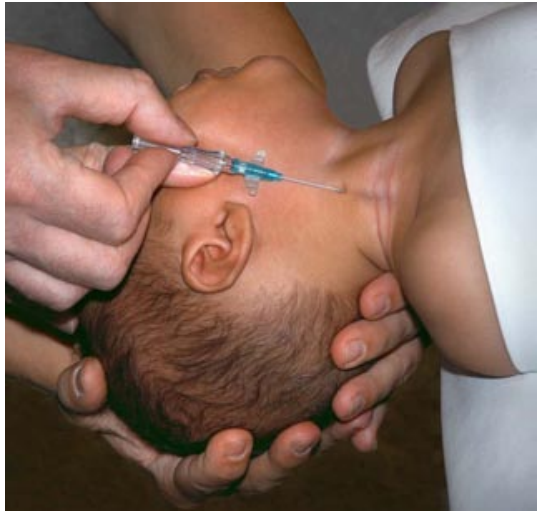

Abb. 3 Optimale Lagerung für die Punktion der V. jugularis externa beim wachen Kind umfasst die Immobilisierung der Arme und des Kopfes durch je eine Hilfsperson. Der Kopf wird dazu über eine Tischkante hyperextendiert und in nach kontralateral rotierter Stellung nach unten gehalten. Die Lagerung ermöglicht das tangentiale Anstechen der externen Jugularvene durch eine Drittperson. Das Schreien des Kindes ist wegen der dadurch entstehenden kurz dauernden Abflussstauung für die Punktion förderlich

ter Verwendung einer Unterlage hängend hyperextendiert und nach kontralateral rotiert (• Abb. 3). Die Punktion erfolgt tangential zur Haut. Beim schreienden Kind wird die Vene durch die entstehende venöse Stauung besser sichtbar und somit einfacher kanülierbar. Mit steigendem Alter wird die Handhabung der externen Jugularvenenpunktion einfacher und gelingt beim anästhesierten Kind in über 80\% [34].

Komplikationen der peripheren Venenkanülierung sind zwar häufig, aber meist harmlos. Lokale oder generalisierte Infektionen, Hämatome und Paravasate werden beschrieben. Nekrosen, v. a. durch paravenös applizierte Barbiturate, Etomidat oder hyperosmolare Lösungen sind möglich [21]. Die akzidentielle Arterienpunktion kommt am häufigsten bei kubitalen Kanülierungsversuchen (A. brachialis) vor, wird aber auch bei der Skalpvenenpunktion (A. temporalis) beschrieben. Um das intraarterielle Injektionsrisiko zu vermindern, empfiehlt es sich, das Gefäß vor jedem Punktionsversuch zu palpieren. Nach erfolgter Punktion soll generell jeder $\mathrm{Zu}$ gang von Hand mit Kochsalz gespült werden. Vereinzelt finden sich auch Fallberichte über Komplikationen der externen Jugularvenenpunktion. Beschrieben werden Infektionen, Hämato-/Pneumothorax, Infusothorax, Venenperforation und Arterien- punktion. Außer den Infektionen treten diese Komplikationen aber ausschließlich bei Versuchen auf, den Katheter über einen Führungsdraht mit J-Spitze zentral zu positionieren.

Allgemein werden vorgängiges Anbringen von EMLA (,eutectic mixture of local anesthetics"), gute Immobilisation der zu stechenden Extremität, Spannen der Haut über der Punktionsstelle, Verwenden kleinkalibriger Kanülen sowie Ruhe, Zeit und Erfahrung von den befragten Anästhesisten als Maßnahmen genannt, die bei schwierigen Punktionsverhältnissen zum Gelingen beitragen. Handwechsel bzw. Hinzuziehen eines in der Kinderanästhesie versierten Kollegen vermögen zusätzlich erfolgversprechend sein.

\section{Intraossärer Zugang}

In den aktuellen Richtlinien des Pediatric Advanced Life Support ist die intraossäre Punktion während der Reanimation immer dann indiziert, wenn innerhalb von 90 s kein anderer vaskulärer Zugang angelegt werden kann [8]. Die Intraossärnadel, angewandt zur Anästhesieeinleitung beim aspirationsgefärdeten Kind, hat aber eine andere Priorität. Lediglich 4 der von uns befragten Anästhesisten haben für diese Indikation je eine Intraossärnadel verwendet. Im Gegensatz zur Praxis betrachten jedoch nahezu die Hälfte der Befragten den intraossären Zugang als primär mögliche Intervention nach missglückten peripheren Punktionsversuchen, wenn aufgrund der klinischen Situation ein vaskulärer $\mathrm{Zu}$ gang erforderlich ist (Fall B). Es ist ein Abwägen zwischen Nutzen und Risiko, wie viele wache Punktionsversuche die Indikation für die Verwendung der Intraossärnadel rechtfertigen.

Besonders geeignet ist die intraossäre Punktion bei Kindern bis zum 6. Lebensjahr, da die Kortikalis des Knochens noch weicher und das Mark stärker vaskularisiert ist [12]. Der Eintritt der Medikamentenwirkung erfolgt ähnlich schnell wie über einen peripheren Venenzugang [26, $38,39]$. Die Einstichstelle bei den o- bis 6Jährigen befindet sich 1-3 $\mathrm{cm}$ distal der Tuberositas tibiae auf der Tibiainnenseite. Der Knochen ist dort oberflächlich tastbar. Nach Desinfektion der Punktionsstelle wird die Nadel epiphysenfern gerichtet und mit Drehbewegungen bis zu einem
Widerstandsverlust eingeführt. Liegt die Nadel richtig, bleibt sie aufrecht stecken. Das Punktionsaspirat kann zur Durchführung von Blutgasanalysen, Blutgruppenbestimmung, Blutkulturen, Elektrolyt- und Hämoglobinbestimmung verwendet werden, dagegen sind Thrombo- und Leukozytenzahl nicht zu verwerten [35]. Andere Möglichkeiten für die intraossäre Punktion sind der distale Femur, der mediale Malleolus, der Humerus und die Spina iliaca anterior superior. Die handelsüblichen Nadeln oder Knochenmarkpunktionsnadeln eignen sich am besten für eine intraossäre Punktion. Da die spontane Tropfgeschwindigkeit intraossär nur ca. $100 \mathrm{ml} / \mathrm{h}$ beträgt, sollte die Flüssigkeit von Hand verabreicht werden. Dabei muss die Einstichstelle beobachtet werden, um eine Paravasion in das den Knochen umgebende Gewebe frühzeitig zu erkennen.

Die prägnantesten Vorteile des intraossären Zugangs liegen in der tiefen Komplikationsrate von weniger als $1 \%$ und der einfachen Handhabung mit einer hohen Erfolgsrate von 94-97\%. Die durchschnittlich benötigte Zeit für das Anlegen des intraossären Zugangs beträgt beim Ungeübten $54 \mathrm{~s}[1,2,16]$.

Neben dem am häufigsten beschriebenen Kompartmentsyndrom kann es in seltenen Fällen zur klinisch vermutlich irrelevanten Fettembolie, zu Frakturen oder Osteomyelitis kommen [10, 24]. Die Inzidenz einer Infektion ist bei sauberer Handhabung und kurzer Verweildauer der Kanüle $(<12 \mathrm{~h})$ vernachlässigbar gering. Kontraindikationen der intraossären Punktion sind Osteogenesis imperfecta, lokale Infekte, Frakturierung oder Vorpunktionen an dem zu punktierenden Knochen.

Nachteile der intraossären Punktion sind in der Invasivität und Schmerzhaftigkeit der Intervention zu sehen. Es existieren kaum Daten zur Praktikabilität des intraossären Zugangs zur Anästhesieeinleitung beim wachen Patienten; insbesondere ist wenig bekannt über die Schmerzhaftigkeit dieses Zugangs. Die Knochenmarkpunktion in Lokalanästhesie ist aber ein gelegentlich praktiziertes Vorgehen auch in der pädiatrischen Onkologie. Anhand eines Falles sehen Stewart u. Kain das elektive Anlegen einer Intraossärnadel bei Kindern als mögliche Intervention, wenn sich die periphervenöse Punktion als extrem 


\section{Hier steht eine Anzeige This is an advertisement}

Springer 
Tabelle 3

Literaturangaben zur Komplikations- und Erfolgsrate von zentralvenösen Zugängen und der externen Jugularvenenpunktion beim anästhesierten Kind

\begin{tabular}{|c|c|c|c|c|}
\hline & V. subclavia & V. jugularis interna & V. femoralis & V. jugularis externa \\
\hline Arterielle Punktion [\%] & $1,5-12,8$ & $1-11,3$ & $10-14$ & Selten \\
\hline Venöse Abflussbehinderung [\%] & 0 & 0 & 14 & kA \\
\hline Hämato-/Pneumothorax [\%] & $1,2-3,1$ & 0 & 0 & Selten \\
\hline Kathetersepsis [\%] & 3 & kA & $0,3-33$ & kA \\
\hline Thrombose [\%] & 0,9 & kA & $11-21$ & kA \\
\hline Fehllage [\%] & 6 & 1,9 & kA & $20-30$ \\
\hline Erfolgsrate [\%] & 86 & 77-97 & $79-86$ & 82 \\
\hline
\end{tabular}

kA keine Angabe.

schwierig erweist [32]. Einige der Befragten sind aufgrund von Einzelbeobachtungen der Meinung, dass die Insertion nach Lokalanästhesie der Haut und des darunter liegenden Periosts dem wachen Kind zugemutet werden kann und nicht schmerzhafter als eine periphere Venenpunktion ist. Zuversichtlich in Bezug auf die Schmerzhaftigkeit wird die Einführung des „bone injection gun" (B.I.G.) betrachtet. Literaturangaben weisen darauf hin, dass nach vorheriger Einstellung der Insertionstiefe die Punktion schneller aber ebenso erfolgreich gelingt wie mit dem konventionellen Verfahren. Doch auch hier fehlen bisher klinische Erfahrungen beim wachen sowie auch beim reanimationspflichtigen Kind [2, 25, 29].

\section{Zentralvenöser Zugang}

Von den befragten Anästhesisten betrachten $21 \%$ nach mehreren misslungenen Versuchen, eine periphere Vene zu kanülieren, die zentrale Venenpunktion als mögliche Intervention, falls ein vaskulärer Zugang aufgrund der klinischen Situation erforderlich ist (Fall B). Dabei würden $50 \%$ die V. femoralis, $40 \%$ die V. subclavia und 10\% die V. jugularis interna wählen.

Mit steigendem Alter ist die Erfolgsrate der zentralvenösen Punktion an jedem Insertionsort zunehmend und liegt bei über 80\% (• Tabelle 3).

Die meist beschrieben Komplikationen der Zentralvenenpunktion der oberen Thoraxapertur sind Hämato- und Pneumotho$\operatorname{rax}[3,6,14,23,27]$; Komplikationen der V.femoralis-Punktion betreffen die akzidentielle Arterienpunktion, Infektionen und Thrombosierung $[4,5,7,17,30,36]$. Die bei- den Letzteren sind erst bei einer Katheterliegedauer von mehr als 5 Tagen klinisch relevant [23]. Generell wird der zentralvenöse Zugang auch bei Kindern von vielen Autoren als sicher betrachtet. Klinisch relevante Komplikationen, die zu einer Verlängerung der Hospitalisation oder erhöhter Morbidität führen, sind selten und korrelieren mit keinem Insertionsort. Es ist jedoch anzumerken, dass weder bei Kindern noch Erwachsenen randomisierte Studien existieren, die die Komplikationsraten der verschiedenen Zugänge unter Notfallbedingungen miteinander vergleichen. Demzufolge kann keiner der 3 zentralen Insertionsorte aufgrund von Literaturangaben in Bezug auf die Komplikationsinzidenz für notfallmäßige Zentralvenenkathetereinlagen als signifikant sicherer eingestuft werden (• Tabelle 3). Obwohl die Hälfte der befragten Anästhesisten den Zugang über die V. subclavia oder die V. jugularis interna wählen, ist anzunehmen, dass deren Punktion beim wachen Säugling und Kleinkind wegen der nicht oder nur mangelhaft vorhandenen Kooperation mit einem höheren Risiko behaftet ist als die Punktion der V. femoralis.

\section{Vorgehen ohne vaskulären Zugang}

Nach weiteren missglückten peripheren Punktionsversuchen betrachten über die Hälfte der befragten Anästhesisten eine intramuskuläre oder inhalative Anästhesieeinleitung beim gesunden, nicht nüchternen Kleinkind (Fall A) als primär mögliches Vorgehen. Dass damit ein erhöhtes Aspirationsrisiko einhergeht, wird wohl immer wieder behauptet, ist aber nicht belegt.
Krauss u. Green zeigen in ihrem Übersichtsartikel, dass seit Einführung von Ketalar in den vergangenen 30 Jahren keine Fälle von ketaminassoziierten Aspirationen bekannt wurden [20]. Somit scheint nach erfolglosen Punktionsversuchen ein alternatives von der Lehrbuchmeinung abweichendes Anästhesieverfahren ohne vaskulären $\mathrm{Zu}$ gang als beste verbleibende Option.

Die Inzidenz der Aspiration ist bei pädiatrischen Patienten mit 0,01-0,1\% selten. Risiken für eine Aspiration stellen ein erhöhter Status in der American-Society-ofAnesthesiologists- (ASA-)Klassifikation, Notfalleingriffe, Anamnese einer durchgemachten Aspiration und Kinder mit gastrointestinalen Missbildungen oder gastroösophagealem Reflux dar [11, 19, 28, 37]. Warner et al. beschreiben das Auftreten beinahe aller beobachteten Aspirationen beim hustenden Kind während der Einleitungsphase und während Manipulationen der Atemwege [37]. Obwohl die Aspirationsgefährdung bei Kindern größer als beim Erwachsenen ist, scheinen schwere Schäden oder gar Todesfälle seltener zu sein. Beim gesunden Kind wird die Aspiration als direkte Todesursache bislang nicht beschrieben $[11,19,28,37]$. Beim Säugling mit Ileus (Fall B) liegen einige Faktoren vor, die eine Aspiration begünstigen: erhöhter ASA-Status, Notfallintervention, Erkrankung des Gastrointestinaltrakts und das Alter selbst. Obwohl sich 50\% aller Aspirationen im Alter zwischen o und 9 Jahren [11] ereignen, würde ein Drittel der Anästhesisten nach mehrmaligen Punktionsversuchen auch bei hoher Aspirationsgefahr eine inhalative oder intramuskuläre Anästhesieeinleitung vornehmen. Die Venenpunktion 
wird nach Anästhesieeinleitung durch die erfolgte Immobilisierung des Kindes und durch die Vasodilatation zwar technisch vereinfacht; der Erfolg ist aber keineswegs garantiert. Die Möglichkeit der Aspiration dauert bei langwierigen Punktionsversuchen bis zur Sicherung der Atemwege an. Bei hoher Aspirationsgefährdung scheint somit das Persistieren auf einen vaskulären Zugang gerechtfertigt.

Ein Abwarten der Nüchternzeit anstelle eines Verfahrens ohne vaskulären $\mathrm{Zu}$ gangs kann sicherlich in Betracht gezogen werden, wenn die klinische Situation ein Verschieben der chirurgischen Intervention zulässt und damit eine Verminderung des Aspirationsrisikos angenommen werden kann. Dass nach erfolgter Nahrungsaufnahme der Zeitfaktor entscheidend für das abnehmende Volumen und den sinkenden Säuregehalt des Magensaftes ist, bleibt unbestritten. Wie lange jedoch bei pädiatrischen Notfällen zugewartet werden soll, ist ein oft diskutiertes Thema in der Literatur. Unter Einwirkung unterschiedlicher Faktoren auf die Magenentleerung lässt sich durch das Abwarten einer festen Nüchternzeit nach durchgemachtem Trauma das Aspirationsrisiko nicht vorhersehbar reduzieren [11, 19, 28, 37]. Das sichere Zeitintervall, das die Aspirationsmortalität und -morbidität signifikant zu reduzieren vermag, ist unbekannt.

\section{Fazit für die Praxis}

Aus den Antworten der durchgeführten Umfrage und dem Literaturstudium lässt sich eine Prioritätenliste für den Umgang mit nicht nüchternen pädiatrischen Notfallpatienten mit schwierigen Venenverhältnissen ableiten. Die intravenöse Blitzeinleitung mit anschließender endotrachealer Intubation wird traditionell als sicherstes Vorgehen bei erfolgtem intravenösen Zugang angesehen. Bei mäBigem Aspirationsrisiko (nicht nüchterner Patient nach Trauma) erscheint insbesondere die intramuskuläre Einleitung mit Ketamin eine gangbare und sichere Alternative. Liegt ein hohes Aspirationsrisko vor (hoher lleus), soll, wenn immer möglich, auf einem vaskulären $\mathrm{Zu}$ gang bestanden werden. Einfache Handhabung und niedrige Komplikationsrate des intraossären Zugangs stehen im Wi- derspruch zur Invasivität und der fehlenden Routine in der Durchführung. In Hinsicht auf die Praktikabilität erscheint der femorale Zugang zur Anästhesieeinleitung von den zentralvenösen Zugängen am zweckdienlichsten. Aufgrund der hohen Erfolgsrate und der einfachen Handhabung ist bei hoher Dringlichkeit und hohem Aspirationsrisiko nach mehreren peripheren Kanülierungsversuchen der intraossäre dem zentralvenösen Zugang vorzuziehen.

\section{Korrespondierender Autor \\ Dr. E.-M. Jordi Ritz}

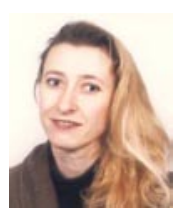

Abteilung Anästhesie, Universitäts-Kinderspital beider Basel (UKBB), Römergasse 8 , 4005 Basel, Schweiz E-Mail: jordie@uhbs.ch

Interessenkonflikt: Der korrespondierende Autor versichert, dass keine Verbindungen mit einer Firma, deren Produkt in dem Artikel genannt ist, oder einer Firma, die ein Konkurrenzprodukt vertreibt, bestehen.

\section{Literatur}

1. Abe KK, Blum GT, Yamamoto LG (2000) Intraosseous is faster and easier than umbilical venous catheterization in newborn emergency vascular access models. Am J Emerg Med 18:126-129

2. Calkins MD, Fitzgerald G, Bentley TB, Burris D (2000) Intraosseous infusion devices: a comparison for potential use in special operations. J Trauma 48:1068-1074

3. Casado-Flores BJ, Barja J, Martino R et al. (2001) Complications of central venous catheterization in critically ill children. Pediatr Crit Care Med 2:57-62

4. Chen KB (2001) Clinical experience of percutaneous femoral venous catheterization in critically ill preterm infants less than 1,000 grams. Anesthesiology 95:637-639

5. Chiang VW, Baskin MN (2000) Uses and complications of central venous catheters inserted in a pediatric emergency department. Pediatr Emerg Care 16:230-232

6. Citak A, Karabocuoglu M, Ucsel R et al. (2002) Central venous catheters in pediatric patients - Subclavian venous approach as the first choice. Pediatr Int 44:83-86

7. Dailey RH (1985) Femoral vein cannulation: a review. J Emerg Med 2:367-372

8. European Resuscitation Council (2000) Part 10: pediatric advanced life support. Resuscitation 46:343-399

9. Finck C, Smith S, Jackson R et al. (2002) Percutaneous subclavian central venous catheterization in children younger than one year of age. Am Surg 68:401-404

10. Fiser D (1990) Intraosseous infusion. N Engl J Med 322:1579-1591
11. Flick RP, Schears GJ, Warner MA (2002) Aspiration in pediatric anesthesia: is there a higher incidence compared with adults? Curr Opin Anaesthesiol 15:323-327

12. Glaeser PW, Hellmich TR, Szewczuga D et al. (1993) Five-year experience in prehospital intraosseous infusions in children and adults. Ann Emerg Med 22:1119-1124

13. Hayashi Y, Uchida O, Takaki O et al. (1992) Internal jugular vein catheterization in infants undergoing cardiovascular surgery: an analysis of the factors influencing successful catheterization. Anesth Analg 74:688-693

14. Johnson EM, Saltzman DA, Suh G et al. (1998) Complications and risks of central venous catheter placement in children. Surgery 124:911-916

15. Kalach N, Barbier C, Kohen R el et al. (2002) Tolerance of nitrous oxide-oxygen sedation for painful procedures in emergency pediatrics: report of $6000 \mathrm{ca}-$ ses. Arch Pediatr 9:1213-1215

16. Jun $\mathrm{H}$, Haruyama AZ, Chang KS, Yamamoto LG (2000) Comparison of a new screw-tipped intraosseous needle versus standard bone marrow aspiration needle for infusion. Am J Emerg Med 18:135139

17. Kanter RK, Zimmerman JJ, Strauss RH et al. (1986) Central venous catheter insertion by femoral vein: safety and effectiveness for the pediatric patient. Pediatrics 77:842-847

18. Kopuz C, Akan H (1996) The importance of the angulation and termination of external jugular vein in central venous catheterization in newborn. Okajimas Folia Anat Jpn 73:155-159

19. Kraus G-B, Pohl B, Reinhold P (1996) Das Kind mit Bagatelltrauma. Anästhesist 45:420-427

20. Krauss B, Green SM (2000) Sedation and analgesia for procedures in children. N Engl J Med 342:938945

21. Lewis G, Hecker JF (1985) Infusion thrombophlebitis. Br J Anaesth 57:220-233

22. Losek JD, Szewczuga D, Glaeser PW (1994) Improved prehospital pediatric ALS care after an EMT-paramedic clinical training course. Am J Emerg Med 12:429-432

23. McGee DC, Gould MK (2003) Preventing complications of central venous catheterization. $\mathrm{N}$ Engl J Med 348:1123-1133

24. Moller JC, Tegtmeyer FK, Schaible TF, Sussmane JB (1996) Intraosseous puncture as vascular access in pediatric emergency and intensive care medicine. Anaesthesiol Reanim 21:103-107

25. Olsen D, Packer BE, Perret J et al. (2002) Evaluation of the bone injection gun as a method for intraosseous cannula placement for fluid therapy in adult dogs. Vet Surg 31:533-540

26. Orlowski JP (1994) Emergency alternatives to intravenous access. Intraosseous, intratracheal, sublingual, and other-site drug administration. Pediatr Clin North Am 41:1183-1199

27. Ruesch $S$, Walder $B$, Tramèr MR (2002) Complications of central venous catheters: internal jugular versus subclavian access - A systematic review. Crit Care Med 30:454-460

28. Splinter WM, Schreiner MS (1999) Preoperative fasting in children. Anesth Analg 87:757-760

29. Spriggs NM, White LJ, Martin SW et al. (2000) Comparison of two intraosseous infusion techniques in an EMT training program. Acad Emerg Med 7:1168

30. Stenzel JP, Green TP, Fuhrman BP et al. (1989) Percutaneous femoral catheterizations: a prospective study of complications. J Pediatr 114:411-415

31. Stern W, Sauer W, Dauber W (1990) Complications of central venous catheterization from an anatomical point of view. Acta Anat (Basel) 138:137-143 
32. Stewart FC, Kain ZN (1992) Intraosseous infusion: elective use in pediatric anesthesia. Anesth Analg 75:626-629

33. Su E, Mann NC, McCall M et al. (1997) Use of resuscitation skills by paramedics caring for critically injured children in Oregon. Prehosp Emerg Care 1:123-127

34. Taylor EA, Mowbray MJ, McLellan I (1992) Central venous access in children via the external jugular vein. Anaesthesia 47:265-266

35. Ummenhofer W, Frei FJ, Urwyler A et al. (1991) Are laboratory values in bone marrow aspirate predictable for venous blood in pediatric patients? Resus citation 27:123-129

36. Wardle SP, Kelsall AWR, Yoxall CW et al. (2001) Percutaneous femoral arterial and venous catheterization during neonatal intensive care. Arch Dis Child Fetal Neonatal Ed 85:F119-122

37. Warner MA, Warner ME, Warner DO et al. (1999) Perioperative pulmonary aspiration in infants and children. Anesthesiology 90:66-71

38. Warren DW, Kissoon N, Mattar A et al. (1994) Pharmacokinetics from multiple intraosseous and peripheral intravenous site injections in normovolemic and hypovolemic pigs. Crit Care Med 22:728-729

39. Wenzel V, Lindner KH, Augenstein S et al. (1999) Intraosseous vasopressin improves coronary perfusion pressure rapidly during cardiopulmonary resuscitation in pigs. Crit Care Med 27:1565-1569

\section{J. R. Brimacombe \\ Laryngeal Mask Anesthesia}

Principles and Practice

W. B. Saunders Company $2004,2^{\text {nd }}$ edn, (ISBN 0-7020-2700-6), 119.00 USD

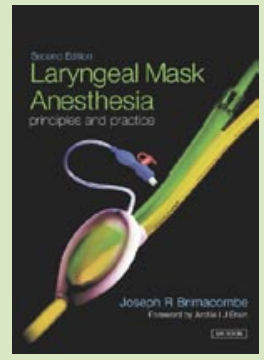

Die von Archie Brain entwickelte Larynxmaske (LMA) hat sich seit über 15 Jahren als Instrument zur Sicherung der Atemwege bewährt. Weltweit wurde sie bis heute bei mehr als 150 Millionen Patienten während Allgemeinanästhesien angewendet. Darüber hinaus ist sie mittlerweile fester Bestandteil der Algorithmen zum Management des schwierigen Atemwegs.

Der Autor, J.R. Brimacombe, ein ausgewiesener Experte mit mehr als 400 Publikationen auf diesem Gebiet, hat es sich mit dem vorliegenden Buch zur Aufgabe gemacht, dem Leser eine umfassende Darstellung des bisherigen Kenntnistandes zum Thema Larynxmaske und anderer extraglottischer Atemwegshilfen zu bieten und ihm gleichzeitig praxisrelevante Empfehlungen zu deren Anwendung an die Hand zu geben. Dazu wurde die gesamte bis zur Drucklegung des Buches zur Verfügung stehende Literatur gesichtet und gewertet.

Das über 600 Seiten umfassende Werk ist in 22 Kapitel aufgeteilt. Nach einem einleitenden historischen Rückblick auf die Entwicklung verschiedener extraglottischer Atemwegshilfen unter besonderer Berücksichtigung der LMA folgen Kapitel, in denen verschiedene Formen der Larynxmaske näher vorgestellt und die anatomischen und pathophysiologischen Grundlagen und Besonderheiten, die bei deren Anwendung zu beachten sind, dargestellt werden. Nachfolgend werden eingehend Indikationen und Kontraindikationen sowie die Vorbereitung und Durchführung einer Anaesthesie unter Verwendung der Larynxmaske behandelt. Weitere Kapitel befassen sich mit den Anwendungsmöglichkeiten der LMA im Rahmen der kardiopulmonalen Reanimation und der Inten- sivtherapie sowie mit dem Stellenwert der Larynxmaske beim Management schwieriger Atemwege. Ausführlich wird auf Besonderheiten im Kindesalter, bei verschiedenen Begleiterkrankungen und speziellen Operationen eingegangen. Weitere Kapitel sind den Sonderformen der LMA gewidmet. Abschließend werden weitere extraglottische Atemwegshilfen beschrieben und deren Bedeutung im Vergleich zur LMA erörtert.

Alle Kapitel sind einheitlich aufgebaut, wobei die Präsentation des Textes aber vielfach von herkömmlichen Lehrbüchern oder Nachschlagewerken abweicht. Auf die Wiedergabe der für die jeweilige Thematik wichtigen Literaturstellen folgt ein wertendes Fazit, bei dem durch die einheitliche Wortwahl der Formulierung (andeuten, darauf schließen lassen, zeigen) zum Ausdruck kommt, in welchem Umfang dieses Statement durch die vorliegende Literatur gestützt wird. Brilliante Abbildungen und instruktive Illustrationen ergänzen den Text und tragen zum besseren Verständnis bei. Am Ende eines jeden Kapitels werden die Ergebnisse wichtiger Metaanalysen aufgeführt und dem eiligen Leser zur Schnellorientierung eine stichwortartige tabellarische Zusammenfassung geboten. Ein angegliedertes umfangreiches Literaturverzeichnis dient als Anregung zur weiteren Vertiefung der zuvor dargestellten Thematik.

Besonders hervorzuheben ist, dass in verschiedenen Kapiteln immer wieder auf mögliche Komplikationen hingewiesen wird und Lösungsvorschläge zu deren Vermeidung gemacht werden, wovon nicht nur Anfänger, sondern auch erfahrene Anwender profitieren können. Wenn überhaupt von einem Nachteil gesprochen werden kann, so ist es die überwältigende Fülle an Einzelinformationen, die dem Leser stellenweise eine hohe Konzentration abverlangt.

Zusammenfassend ist das Buch ein ideales Nachschlagewerk zu allen Aspekten im Umgang mit der Larynxmaske und sollte daher in keiner anästhesiologischen Fachbibliothek fehlen.

U. Grundmann (Homburg/Saar) 